Editor's Note: These short, critical reviews of recent papers in the Journal, written exclusively by graduate students or postdoctoral fellows, are intended to summarize the important findings of the paper and provide additional insight and commentary. For more information on the format and purpose of the Journal Club, please see http://www.jneurosci.org/misc/ifa_features.shtml.

\title{
Action-Coding Neurons in Primary Motor Cortex: Making Sense of M1 Activity during Action Perception
}

\author{
Jean-François Lepage, Mélissa Lortie, and François Champoux \\ Centre de Recherche de l'Hôpital Sainte-Justine, Montréal, Québec, Canada H3T 1C5 \\ Review of Tkach et al. (http://www.jneurosci.org/cgi/content/full/27/48/13241)
}

In the last decade, considerable efforts have been devoted to the description of motor resonance, a process by which observation of a goal-directed action triggers brain activation highly similar to that associated with the execution of the same action. The seminal discovery at the root of the resurgent interest in the neural mechanism underlying motor resonance was the description of mirror neurons in area F5 of the macaque monkey. Mirror neurons discharge when an individual performs and/or sees an action (see Rizzolatti and Craighero, 2004), and they are part of a complex frontoparietal "mirror" network that includes area F5 of the ventral premotor cortex and area PF of the inferior parietal lobule.

Until now, the primary motor cortex (M1), despite its crucial role in motor performance, was not considered to be part of this mirroring system, because it was not thought to respond to the passive observation of actions. In a study recently published in The Journal of Neuroscience, however, Tkach et al. (2007) provide the first demonstration of the existence of single neurons in monkey M1 that behave

Received Dec. 7, 2007; revised Jan. 11, 2008; accepted Jan. 13, 2008.

J.-F.L. and F.C. are supported by the Canadian Institutes of Health Research, and M.L. is supported by the Fonds de Recherche en Santé du Québec.

Correspondence should be addressed to Jean-François Lepage, Département de Recherche, Sainte-Justine Hospital Research Center, 3175 Ch. Côte-Sainte-Catherine, Montréal, Québec, Canada H3T 1C5. E-mail: jf.lepage@umontreal.ca.

DOI:10.1523/JNEUROSCI.5422-07.2008

Copyright $\odot$ 2008 Society for Neuroscience $\quad 0270-6474 / 08 / 281995-02 \$ 15.00 / 0$ like mirror neurons. The authors recorded single-neuron activity in M1 during a task requiring trained monkeys to guide a cursor to a target location using a robotic manipulandum. Subsequently, the animals passively watched a replay of the cursor-to-target action sequence. Results showed that M1 neurons encode a considerable amount of information regarding action production that was also present during the passive observation of the action sequence, such as sensitivity to preferred direction and oscillatory power modulation. Spike responses clearly demonstrated that the observation of cursor movement toward the target modulates neural activity in the same way as the execution task [Tkach et al. (2007), their Fig. 2 (http://www.jneurosci.org/cgi/content/ full/27/48/13241/F2)]: many neurons that showed increases or decreases in mean firing rate displayed similar profile during action observation. Importantly, control tasks revealed that the presence of a target during observation was necessary for M1 neurons to display firing patterns similar to those elicited by execution of the task.

An interesting, albeit briefly discussed, aspect of the data is the analysis of oscillatory power, which allows comparisons between local field potentials recorded in monkeys and scalp EEG recordings in humans. It is difficult, from the data shown, to draw firm conclusions regarding the correspondence between beta band $(10-25 \mathrm{~Hz})$ activity reported in the Tkach et al. [2007; their Fig. 5 (http://www. jneurosci.org/cgi/content/full/27/48/ 13241/F5)] study and human EEG findings. Integrated power during all observation conditions, including the simultaneous observation of cursor and target, was larger than during the active movement condition. It is unclear, however, whether observation of the target or cursor movement alone produced significantly different beta activity than cursorto-target interactions, a pattern that would appear to be necessary to replicate human EEG findings (Muthukumaraswamy and Johnson, 2004). This inconsistency is probably attributable to the large frequency band $(10-25 \mathrm{~Hz})$ that was selected for analysis. Indeed, human studies have consistently showed decreased spectral power during execution and observation of action in relatively narrow and specific frequency bands [EEG, 8-12 $\mathrm{Hz}$ (Muthukumaraswamy and Johnson, 2004); MEG, $\approx 20 \mathrm{~Hz}$ (Hari, 2006)].

Traditionally, mirror neurons properties have been studied in situations in which monkeys observe biological movement performed by a human model or conspecific (typically object-oriented grasping). Hence, a novel aspect of the Tkach et al. (2007) experiment was the use of an inanimate effector to execute the action. Interestingly, the authors argue that such a design prevents the classification of M1 cells as mirror neurons because of the absence of an object-effector interaction. It is unclear why this should the case because, for example, visual perception of an 
action is not necessary for mirror neurons to fire. Indeed, Umiltà et al. (2001) have shown that a mental representation of what the observed action means is sufficient to elicit activation of mirror neurons circuits. They showed that even when the final part of the grasping movement was hidden, and could hence only be inferred, some mirror neurons still responded, implying that an internally generated motor representation was sufficient to trigger a response. In the present case, we would suggest that a mirror neuron designation is justified because (1) the cells respond to both the execution and observation of the results of an action; and (2) a clear interaction between the action and its goal (the target) is necessary for congruent activity to occur in M1. It is, however, clear that confirmation of M1 spiking patterns during observation and execution of naturalistic biological actions would considerably strengthen the contention that mirror neurons are indeed found in primary motor cortex.

Congruent patterns of activity during execution and observation of action have been measured in human primary motor cortex, most notably with transcranial magnetic stimulation (Fadiga et al., 2005), but until now, the origin of this activity remained elusive. It had been suggested that M1 corticospinal facilitation result- ing from passive observation of biological actions was a consequence of the corticocortical connections originating in mirror neuron-rich premotor cortex (Fadiga et al., 2005). Although it is probably true that frontal mirror neuron areas exert a modulatory influence on M1 excitability during action observation, it now appears likely that at least some of this activity is attributable to a local, active motormatching process. Temporally sensitive imaging methods such as MEG can offer insight into the time course of this activity while at the same time providing important clues about the specific role of M1 in the complex chain of neural events that underlies action understanding. Indeed, Nishitani and Hari (2000) have shown that activity in human primary motor cortex during observation of hand actions occurs later than inferior frontal gyrus activation (presumably the homolog of monkey F5). This suggests that the contribution of M1 to the understanding of others' actions may be near the end of the activation sequence that accompanies action observation. Although additional studies are needed to pinpoint the exact role of M1 in this complex process, especially at the single-neuron level, it is tempting to speculate that specific information about the dynamics of effector selection and use when observing a conspe- cific performing an action is computed in the newly discovered cells of Tkach et al. (2007). Finally, these new data show that exciting times are ahead for M1 research, and only time will tell what new functions (going beyond simple motor command) will end up being associated with primary motor cortex.

\section{References}

Fadiga L, Craighero L, Olivier E (2005) Human motor cortex excitability during the perception of others' action. Curr Opin Neurobiol 15:213-218.

Hari R (2006) Action-perception connection and the cortical mu rhythm. Prog Brain Res 159:253-260.

Muthukumaraswamy SD, Johnson BW (2004) Changes in rolandic mu rhythm during observation of a precision grip. Psychophysiology 41:152-156.

Nishitani N, Hari R (2000) Temporal dynamics of cortical representation for action. Proc Natl Acad Sci USA 97:913-918.

Rizzolatti G, Craighero L (2004) The mirrorneuron system. Annu Rev Neurosci 27:169-192.

Tkach D, Reimer J, Hatsopoulos NG (2007) Congruent activity during action and action observation in motor cortex. J Neurosci 27:13241-13250.

Umiltà MA, Kohler E, Gallese V, Fogassi L, Fadiga L, Keysers C, Rizzolatti G (2001) I know what you are doing. A neurophysiological study. Neuron 31:155-165. 